\title{
Distracted by danger: Temporal and spatial dynamics of visual selection in the presence of threat
}

\author{
Manon Mulckhuyse $^{1} \cdot$ Edwin S. Dalmaijer $^{2}$
}

Published online: 6 November 2015

(C) Psychonomic Society, Inc. 2015

\begin{abstract}
Threatening stimuli are known to influence attentional and visual processes in order to prioritize selection. For example, previous research showed faster detection of threatening relative to nonthreatening stimuli. This has led to the proposal that threatening stimuli are prioritized automatically via a rapid subcortical route. However, in most studies, the threatening stimulus is always to some extent task relevant. Therefore, it is still unclear if threatening stimuli are automatically prioritized by the visual system. We used the additional singleton paradigm with task-irrelevant fear-conditioned distractors (CS+ and CS-) and indexed the time course of eye movement behavior. The results demonstrate automatic prioritization of threat. First, mean latency of saccades directed to the neutral target was increased in the presence of a threatening $(\mathrm{CS}+)$ relative to a nonthreatening distractor (CS-), indicating exogenous attentional capture and delayed disengagement of covert attention. Second, more error saccades were directed to the threatening than to the nonthreatening distractor, indicating a modulation of automatically driven saccades. Nevertheless, cumulative distributions of the saccade latencies showed no modulation of threat for the fastest goal-driven saccades, and threat did not affect the latency of the error saccades to the distractors. Together these results suggest that threatening stimuli are automatically prioritized in attentional and visual selection but not via faster
\end{abstract}

Manon Mulckhuyse

manon.mulckhuyse@gmail.com

1 Donders Institute for Brain, Cognition and Behavior, Affective Neuroscience, Radboud University, Kapittelweg 29, Nijmegen, The Netherlands

2 Department of Experimental Psychology, University of Oxford, Oxford, UK processing. Rather, we suggest that prioritization results from an enhanced representation of the threatening stimulus in the oculomotor system, which drives attentional and visual selection. The current findings are interpreted in terms of a neurobiological model of saccade programming.

Keywords Attention - Emotion - Eye movement . Oculomotor system $\cdot$ Fear conditioning

Once you're bitten by a dog, your perception of dogs may be biased forever: You will probably have the feeling that there are more dogs today than ever before and often you will note a dog before anyone else does. In other words, you prioritize information that is threatening to you. How the visual system prioritizes threatening information is still an ongoing debate. For example, "the direct route" theory suggests that visual threatening information is processed automatically via a specialized subcortical pathway (De Gelder, Van Honk, \& Tamietto, 2011; LeDoux, 2000; Liddell et al., 2005; Öhman, Flykt, \& Esteves, 2001). This pathway processes visual information directly from the retina to the superior colliculus and via the pulvinar on to the amygdala. According to this theory, threatening information is processed not only automatically but also faster than neutral or nonthreatening information. However, the "multiple waves model" suggested by Pessoa and Adolphs (2010) disputes the existence of a direct subcortical route via the pulvinar to the amygdala for visual processing. Their theory suggests that prioritization is accomplished by modulating interactions between subcortical and cortical connections that, in addition, do not process emotional visual information any faster than other salient visual information. Nevertheless, previous research on emotional covert attention indicates that threatening stimuli are detected faster and therefore possibly processed faster than nonthreatening stimuli 
(e.g., Hansen \& Hansen, 1988; Öhman, Flykt, \& Esteves, 2001; Öhman, Lundqvist, \& Esteves, 2001). However, in these studies the emotional stimulus is always to some extent task relevant and therefore part of the top-down goal or task set of the participant. Consequently, these studies do not convey information about automatic or bottom-up driven prioritization of emotional stimuli in visual selection (see, for reviews on automatic emotional attention, Carretié, 2014; on bottom-up control, Theeuwes, 2010).

A recent study by Schmidt, Belopolsky, and Theeuwes (2014; see also Notebaert, Crombez, Van Damme, De Houwer, \& Theeuwes, 2011) demonstrated emotional modulation of exogenous attention in a visual search task with fearconditioned but task-irrelevant distractor stimuli. The results showed that a threatening distractor slowed search more than an equally visually salient but nonthreatening distractor. Although these results indicate a bottom-up driven modulation of attentional selection, they do not convey information about how the threatening stimulus is prioritized; for example, whether the threatening distractor captured attention faster or whether the threatening distractor held attention longer relative to the nonthreatening distractor.

The aim of the current study was to examine how threat modulates automatically driven processes in visual selection, such as attentional and oculomotor capture. For this, we modified the additional singleton paradigm (Godijn \& Theeuwes, 2002; Theeuwes, Kramer, Hahn, \& Irwin, 1998) and indexed the time course of eye-movement behavior. Eye movements are a direct and very sensitive measure of visual selection, as they can occur as early as $150 \mathrm{~ms}$ after stimulus onset, while manual responses typically vary between 300 and $2000 \mathrm{~ms}$. Therefore, eye movements can reveal emotional modulation of visual selection not visible in manual behavior, such as manual reaction time or accuracy (e.g., Bannerman, Milders, de Gelder, \& Sahraie, 2009; Bannerman, Milders, \& Sahraie, 2009, 2010a).

A number of previous studies on emotional modulation of eye-movement behavior found evidence for faster detection of emotional stimuli (e.g., Bannerman, Milders, de Gelder, \& Sahraie, 2009; Bannerman, Milders, \& Sahraiem, 2009; Kissler \& Keil, 2008; LoBue, Matthews, Harvey, \& Stark, 2014). For example, Bannerman, Milders, de Gelder, \& Sahraie(2009) and Bannerman, Milders, and Sahraie (2009) showed a decrease in saccade latency when a saccade is directed to the location of a threatening stimulus relative to a nonthreatening stimulus. Other studies found evidence for longer fixation durations on emotional relative to neutral stimuli in free-viewing paradigms (e.g., Calvo \& Lang, 2004; Humphrey, Underwood, \& Lambert, 2012; Lang, Greenwald, Bradley, \& Hamm, 1993), suggesting delayed disengagement from an emotional stimulus (Fox, Russo, Bowles, \& Dutton, 2001; Fox, Russo, \& Dutton, 2002). However, similar to the covert attention studies discussed before, most eye movement studies presented emotional stimuli that were always to some extent task relevant. For example, observers were instructed to saccade toward or away from the emotional stimulus (e.g., Bannerman, Milders, de Gelder, \& Sahraie, 2009; Bannerman, Milders, \& Sahraie, 2009; Kissler \& Keil, 2008; Nummenmaa, Hyönä, \& Calvo, 2006) or to assess the valence, category or memory of presented pictures (Calvo \& Lang, 2004; Humphrey et al., 2012; Lang et al., 1993; LoBue et al., 2014). Therefore, observers had to process and attend to the emotional stimulus in order to perform the task. In other words, the emotional stimulus was part of their top-down goal or task set. Task set has a major influence on search behavior and reaction times (Yantis, 1993). For example, a study by Hunt, Cooper, Hungr, and Kingstone (2007) demonstrated emotional modulation of eye-movement behavior only when subjects had to search for an emotional target stimulus, indicating that the emotional stimulus has to be part of a top-down goal or task set in order to affect visual selection.

In the current study, we investigated the influence of a task irrelevant threatening distractor on the oculomotor system with the additional singleton paradigm. With this paradigm it is possible to measure covert attention and overt attention processes because the visually salient distractor typically captures attention and sometimes the eyes. Note that the distractor in this paradigm is completely task irrelevant and therefore evokes involuntary or bottom-up driven capture (Theeuwes et al., 1998).

In the task, participants are presented with a search array consisting of several elements displayed on an imaginary circle. Participants are asked to make a speeded saccade to a target stimulus that is less visually salient than the additional singleton, the distractor. The distractor is a sudden onset and presented simultaneously with the target. Typically, saccades directed to the target are slower in the presence of the distractor. This increase in saccade latency is explained by an initial shift of covert attention (attentional capture) to the salient distractor before attention moves on to the less salient target, and, subsequently, a correct saccade can be programmed and executed. Note that this explanation assumes the tight coupling between a shift of covert attention and subsequent oculomotor programming as argued in the premotor theory (Rizzolatti, Riggio, Dascola, \& Umilta, 1987; but see Belopolsky \& Theeuwes, 2012). In addition, with this paradigm it is typically found that in a subset of the distractorpresent trials, initial eye movements are first directed to the distractor instead of to the target. These error saccades, named oculomotor capture, are usually very fast and are assumed to be automatic because they are driven by stimulus properties and not by any top-down goals (Godijn \& Theeuwes, 2002; Mulckhuyse et al., 2008).

In addition, we used distributions of saccadic latency to examine influences of threat on both fast and slower saccadic responses because previous research has shown that the 
latency of a saccade influences saccadic behavior (Godijn \& Theeuwes, 2002; McSorley, Haggard, \& Walker, 2006). For instance, a task-irrelevant distractor next to the saccadic path induces a deviation of the saccade path toward the distractor when a saccade is initiated shortly after target onset (short latency saccade), whereas the same distractor induces a deviation away from the distractor when a saccade is initiated after around 200 milliseconds or more (long latency saccade) after target onset (McSorley et al., 2006). Moreover it was shown that these saccade deviations (toward and away from a distractor) are both modulated by emotion (Mulckhuyse, Crombez, \& Van der Stigchel, 2013; Schmidt, Belopolsky, $\&$ Theeuwes, 2012). Furthermore, previous research has also shown that saccade latency affects end point. Short latency saccades are more often driven by salient visual stimuli (oculomotor capture) than are long latency saccades (e.g., Godijn \& Theeuwes, 2002; Mulckhuyse, Van Zoest, \& Theeuwes, 2008). In general, it is assumed that stimulus-driven information, such as the visual saliency of a stimulus, affects short latency saccades, which, for example, can result in more oculomotor capture for short latency saccades relative to long latency saccades (Mulckhuyse et al., 2008). We were interested whether threat would affect saccadic behavior of short as well as long latency saccades.

Furthermore, we used a differential fear-conditioning procedure (Mackintosh, 1983) to present a threatening (CS+) and nonthreatening (CS-) distractor stimulus. Fear-conditioning has proven to be an extremely robust and precise experimental approach for studying fear in animals as well as in humans (e.g., Brown, Kalish, \& Farber, 1951; Delgado, Olsson, \& Phelps, 2006; LeDoux, 2003; Maren, 2001;

Pineles, Orr, \& Orr, 2009; Sehlmeyer et al., 2009). In differential fear-conditioning, one biologically neutral stimulus (CS) is associated with a noxious or aversive unconditioned stimulus (US) by presenting it in close temporal proximity (LeDoux, 2014). Subsequently, the CS+ predicts the possible aversive event and hence becomes a threatening stimulus. The CS- (another biologically neutral stimulus) is not associated with the US and therefore becomes a nonthreatening stimulus (Lissek et al., 2008).

A fear-conditioned stimulus differs in several respects from a threatening picture. First, the CS+ serves as a cue for a possible aversive event to the participant, whereas a threatening picture of a snake, for example, is a symbolic representation of that threat. Second, the visual features between the $\mathrm{CS}+$ and CS- distractor are more easily controlled for (colors are counterbalanced between subjects), whereas visual features between threatening and nonthreatening pictures are sometimes more difficult to control for (e.g., Calvo \& Nummenmaa, 2008; Hunt et al., 2007; Tipples, Young, Quinlan, Broks, \& Ellis, 2002).

We expected to find more oculomotor capture by the threatening distractor $(\mathrm{CS}+)$ than the nonthreatening distractor
(CS-), and we expected to find a decrease in latency of saccades directed to the threatening distractor compared to the nonthreatening distractor. In addition, we expected to find an increase in latency of correct saccades in the presence of the threatening distractor relative to the nonthreatening distractor for fast as well as slow saccades.

\section{Method}

\section{Participants}

Sixteen undergraduate students (11 female, ages between 19 and 33) participated to fulfill course requirements or they were paid 10 euro. All participants provided informed consent before participation and were free to terminate the experiment at any time. Participants had normal or corrected-to-normal vision. The medical ethical committee of the University Medical Center Utrecht approved the study.

\section{Apparatus and design}

Participants were seated $65 \mathrm{~cm}$ from a Phillips Brilliance $202 \mathrm{P} 7$ (running at $60 \mathrm{~Hz}$, with a resolution of $1,024 \times 768$ ) with their head positioned on a chin rest. Eye movements were registered by means of a video-based eye tracker (EyeLink 1000, by SR Research Ltd., Canada), with a $1000 \mathrm{~Hz}$ temporal and a $0.025^{\circ}$ spatial resolution. Participants fixated a central fixation cross on a black background (see Fig. 1). The stimulus display consisted of six filled light gray $(x=0.291, y=$ $0.318,21 \mathrm{~cd} / \mathrm{m}^{2}$ ) circles, each $1.5^{\circ}$ in diameter that were equally spaced around the fixation point on an imaginary circle with a radius of $9.8^{\circ}$. In each circle, a small black cross $\left(1.2^{\circ}\right.$ in diameter) was presented. The circles were presented at $1,3,5,7,9$, and 11 o'clock with an angular separation of $60^{\circ}$ between the circles. In the distractor conditions, a filled circle, $1.5^{\circ}$ in diameter, was presented on the imaginary circle exactly between two filled gray circles. The stimuli could be either red $(x=0.548, y=0.398)$ or green $(x=0.299, y=0.559)$. The red and green colors were matched for physical luminance $(28.4 \mathrm{~cd} / \mathrm{m} 2)$ and counterbalanced between subjects. The experiment was programmed in Python, using the PyGaze toolbox (Dalmaijer, Mathôt, \& Van der Stigchel, 2014).

Figure 1 shows the sequence of a trial in all conditions. Each trial began with a white plus sign. The gray circles with the black crosses were presented for at least $1,000 \mathrm{~ms}$ with an additional random jitter between $0 \mathrm{~ms}$ and $300 \mathrm{~ms}$. In five of the six circles, the black cross disappeared. The cross that stayed on the screen indicated the target location. Simultaneously with the offsets of the black crosses, the fixation point disappeared, and in the distractor conditions, the additional filled circle was presented. The target appeared equally often at each of the four possible target locations: $1,5,7$, or 11 


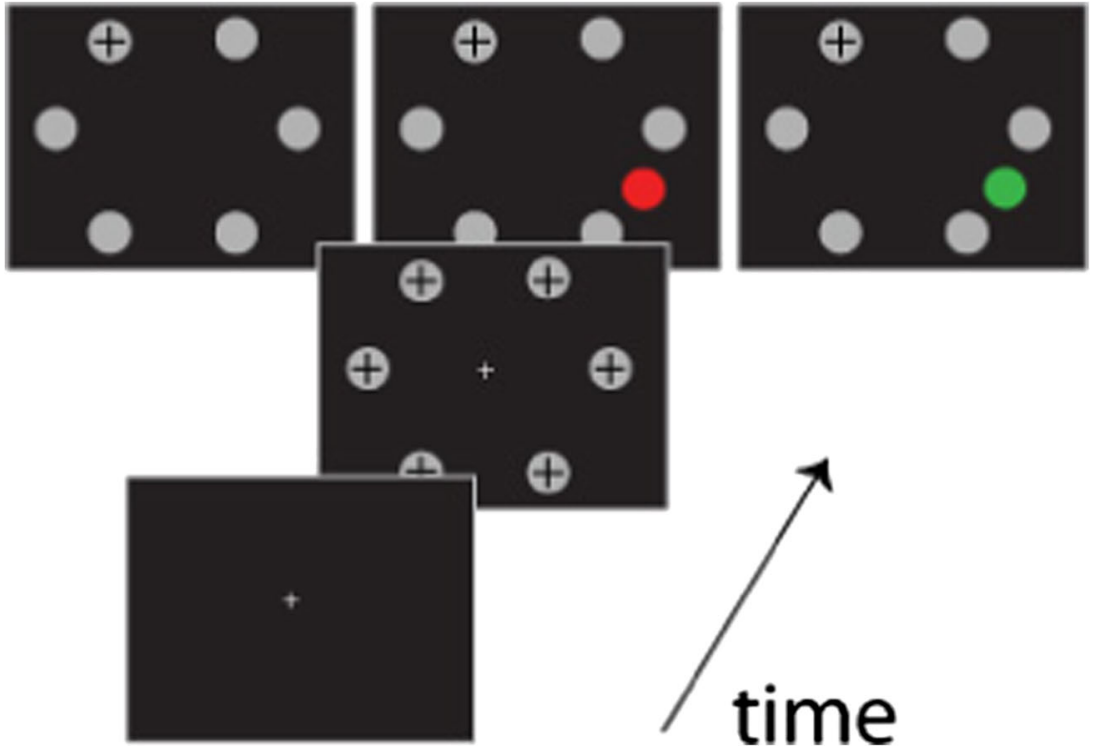

Fig. 1 From bottom to top, a sequence of events in a trial with, on the left panel, the distractor absent condition, in the middle and on the right, a CS+ and a CS- distractor trial or vice versa (the colors were counterbalanced between subjects). (Color figure online.)

o'clock. The distractor could appear at 2, 4, 8, or 10 o'clock, but always with an angular separation of $90^{\circ}$ or $150^{\circ}$ between distractor and target.

\section{Procedure}

Before the experiment, a differential fear conditioning procedure was started in which participants viewed the distractors one by one while one of the colors (Conditioned Stimulus: $\mathrm{CS}+$ ) was paired with an aversive noise (Unconditioned Stimulus: US) whereas the other color (CS-) was not (Mackintosh, 1983). Eight participants were presented with a red CS+ and a green CS- and eight participants vice versa. Due to this method, a comparison between the two conditions was not confounded by potential differences in visual features between stimuli (e.g., Calvo \& Nummenmaa, 2008; Hunt et al., 2007). The US consisted of a $200 \mathrm{~ms} 100 \mathrm{~dB}$ white noise that ended with the offset of the CS+. If participants were able to correctly identify the color that predicted the noise, the experiment started. In the experiment, participants were required to make a speeded saccade to the target while ignoring the presence of a distractor. Each participant performed nine blocks of each 48 trials. In one block the distractor was absent; in the other eight blocks distractor presence was mixed (CS+ and CS- distractor). The order of blocks was randomly generated. In addition, between $5 \%$ and $10 \%$ reinforcement trials (in which the CS+ was presented together with the US) were added to each block to avoid habituation and extinction of fear (for a review, see Hofmann, 2008). The reinforcement trials were randomly mixed with the experimental trials and not further analyzed.

Participants rated their anxiety level three times on a visual analogue scale (VAS): before the experiment started, during the experiment (after four blocks), and when the experiment ended. Subsequently, ratings of fear of the CS+ and CS- and ratings of the intensity, unpleasantness, and expectancy of the US were obtained using Likert scales $(1=$ not at all to $9=$ extremely $)$.

\section{Results}

\section{Anxiety rating}

Mean rating on the VAS (on a scale from 0 to 100) before the experiment started was $14.2(S D=19.0)$, during the experiment $36.3(S D=35.5)$, and when the experiment ended 26.6 $(S D=27.7)$. A repeated measures ANOVA on VAS rating showed a main effect, $F(2,30)=9.75, p<.05, \eta_{\mathrm{p}}{ }^{2}=0.39$, due to enhanced anxiety during the experiment relative to before $(p<.01, d=1.08)$ and relative to after the experiment $(p<.05, d=0.6)$.

\section{Fear conditioning}

All participants successfully learned the CS-US contingencies at the end of the acquisition phase. Mean ratings of intensity, unpleasantness, and fear of the US were $6.7(S D=1.7), 6.8(S D$ $=2.3)$, and $5.1(S D=3.0)$, respectively. As the ratings were not normally distributed, nonparametric tests were employed. Participants rated fear of the CS+ $(M=4.2, S D=2.9)$ higher than of the CS- $(M=1.6, S D=1.0$; Wilcoxon signed-rank test, $Z=$ 2.94, $N$-ties $=5, p<.01)$. Furthermore, US expectancy ratings for the CS+ $(M=4.4, S D=2.1)$ were higher than for the CS- $(M$ $=1.9, S D=1.8$; Wilcoxon signed-rank test, $Z=2.6, N$-ties $=1$, $p<.01)$. US expectancy rating has demonstrated to be a valuable measurement method in fear conditioning (Boddez et al., 
2013). Therefore, these results suggest that our fear conditioning procedure was successful.

\section{Eye movement behavior}

The initial saccade was assigned to a target or a distractor if the start point of the saccade was within $3^{\circ}$ of the fixation point and when the endpoint was within $3^{\circ}$ of the center of the target or distractor position (maximum angular separation of $17.5^{\circ}$ from the center of the target or distractor). This cutoff corresponds to roughly half the distance between target/distractor and the nearest placeholder. If the endpoint was beyond the assigned area of the target and distractor, it was assigned to one of the other placeholders in the display and defined as "elsewhere than the target or the distractor." Trials for which initial saccade latencies were below $80 \mathrm{~ms}$, above $600 \mathrm{~ms}$, or for which no saccade was detected were excluded from analysis ( $5.5 \%$ of all trials).

\section{Saccades to the target}

In the distractor-absent condition, $72.6 \%(S E=2.9)$ of all initial saccades went to the target, in the CS- distractor condition $53.0 \%(S E=2.9)$, and in the $\mathrm{CS}+$ distractor condition, $47.8 \%(S E=4.1$; see Fig. 2). Nonparametric tests were employed for the percentage scores, because the data were not normally distributed. A Friedman test on percentage correct saccades showed a significant main effect of distractor condition, $\chi^{2}(2, N=16)=7.13, p<.01, W=0.22$. Subsequent Wilcoxon signed-rank tests showed that significantly more initial saccades were directed to the target when no distractor was presented than when the CS- distractor was presented $(Z=$ 3.46, $N$-ties $=0, p<.01, r=0.42)$ or the CS+ distractor was presented $(Z=3.46, N$-ties $=0, p<.01, r=0.45)$. In addition, significantly more initial saccades were directed to the target when a CS- distractor was presented relative to a CS+ distractor $(Z=2.02, N$-ties $=0, p<.05, r=0.86)$.

An ANOVA on mean latency for saccades correctly directed to the target showed that there was a significant main effect of distractor, $F(2,30)=4.15, p<.05, \eta_{\mathrm{p}}^{2}=2.2$. Planned comparisons showed that the mean saccade latency in the $\mathrm{CS}+$ distractor condition $(271 \mathrm{~ms}, S E=10 \mathrm{~ms})$ was significantly longer than the mean saccade latency in the CSdistractor condition $(263 \mathrm{~ms}, S E=9 \mathrm{~ms}) t(15)=2.17, p<$ $.05, d=0.54$, and significantly longer than the mean saccade latency in the distractor absent condition $(257 \mathrm{~ms}, S E=10 \mathrm{~ms})$ $t(15)=2.51, p<.05, d=0.56$. These results indicate that the $\mathrm{CS}+$ distractor captured and held covert attention before a saccade was executed to the target. Furthermore, the mean saccade latency in the CS- distractor condition did not differ from the distractor absent condition $(p=.26)$.

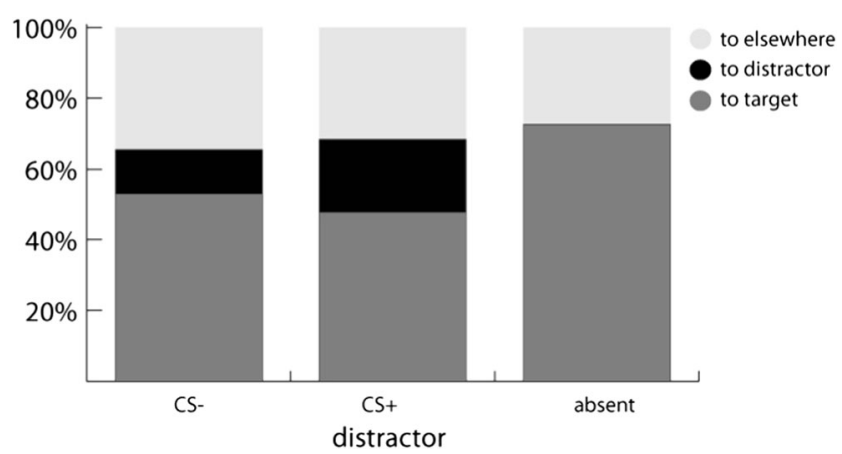

Fig. 2 The proportion of saccades directed toward the target (dark gray), toward the distractor (black), or toward elsewhere (light gray) in the display is shown for all conditions: on the left when the CS- distractor is presented, in the middle when the CS+ distractor is presented, and on the right when no distractor is presented

\section{Time course of saccades to the target}

To examine the effect of threat on the time course of saccades directed to the target, we calculated individual cumulative distribution functions of the saccade latencies for each subject and each condition. These were averaged in four bins using the Vincentizing procedure (Ratcliff, 1979). Figure 3 shows the distributions of the saccade latencies in the three conditions. On the left, mean latency of fast saccades (Bin 1), and on the right, mean latency of the slowest saccades (Bin 4) are depicted.

An ANOVA on mean saccade latency with bin (1, 2, 3, and 4 ) and distractor (CS+, CS-, and absent) as factors revealed a main effect of bin, $F(3,45)=188.02, p<.01, \eta^{2}=0.93$, with Greenhouse-Geisser correction, a main effect of distractor, $F(2,30)=4.26, p<.05, \eta_{\mathrm{p}}{ }^{2}=0.22$, and an interaction between bin and distractor, $F(6,90)=3.15, p<.05, \eta_{\mathrm{p}}{ }^{2}=0.17$, with Greenhouse-Geisser correction. Planned comparisons showed that saccade latency of correct saccades in the presence of a CS+ distractor was increased relative to no distractor in the second, the third, and the fourth bin (all $p \mathrm{~s}<.05$, onetailed). Furthermore, saccade latency of correct saccades in the presence of a CS+ distractor was increased relative to a CSdistractor in the second, the third, and the fourth bin (all $p \mathrm{~s} \leqq$ .05 , one-tailed). The increase in saccade latency suggests attentional capture by the distractor. There was no significant difference in the first bin between the three conditions ( $p>$ .18 ), indicating that in these trials, covert attention was not first shifted to the distractor stimulus.

\section{Error saccades to the distractor}

In the CS- distractor condition, $12.5 \%(S E=2.9)$ of all initial saccades went to the distractor, and in the CS+ distractor condition, $20.6 \%$ ( $S E=3.74$; see Fig. 2). A Wilcoxon signed-rank test showed a significant effect between the two conditions ( $Z$ 


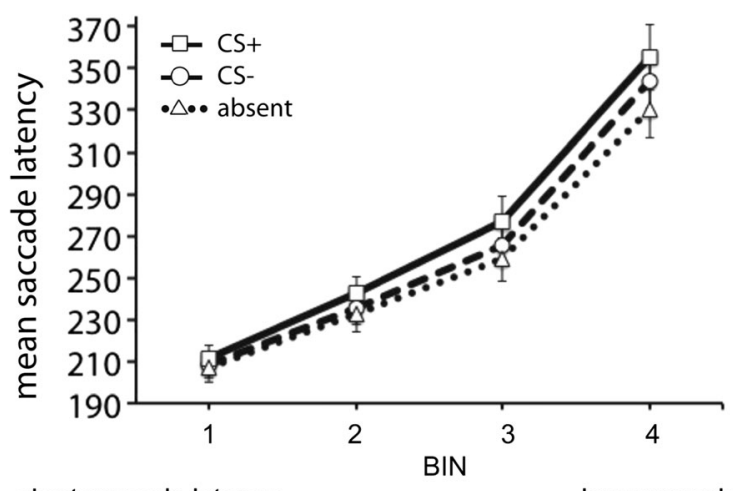

long saccade latency
Table 1 Mean saccade latency (and standard errors) for each distractor condition (CS+ and CS-) and for each bin (1,2, and 3)

\begin{tabular}{lll}
\hline Bin & Color singleton & \\
\cline { 2 - 3 } & CS + & CS- \\
\hline 1 & $170 \mathrm{~ms}(2 \mathrm{~ms})$ & $173 \mathrm{~ms}(3 \mathrm{~ms})$ \\
2 & $189 \mathrm{~ms}(5 \mathrm{~ms})$ & $188 \mathrm{~ms}(5 \mathrm{~ms})$ \\
3 & $226 \mathrm{~ms}(9 \mathrm{~ms})$ & $218 \mathrm{~ms}(7 \mathrm{~ms})$ \\
\hline
\end{tabular}

relative to the CS- distractor could be that these saccades are not driven by the threatening information at that specific location but rather are driven by a general enhanced state of anxiety. If the latter instance would be the case, we would also expect to find more error trials directed elsewhere than to the distractor when the CS+ distractor was presented relative to when the CS- distractor was presented. As can be seen in Fig. 2, this is not what the results show. In contrast, the results show that participants made more error saccades directed elsewhere when a CS- distractor was presented $(34.5 \%, S E=3)$ than when a CS+ distractor was presented $(31.6 \%, S E=3 ; Z=$ 2.27, $N$-ties $=1, p<.05, r=0.93$ ). This finding indicates that the higher percentage of error saccades directed to the CS+ distractor was caused by the information at that location and was not the result of a nonspecific effect induced by the threatening distractor.

Furthermore, to determine that the latency of the error saccades directed to the distractors were indeed affected by the information at that location and not by a "general state" induced by the distracters (either CS- or CS+), we performed related samples $t$ tests on saccade latency between error saccades directed to the distractor and error saccades directed elsewhere. We performed this test for each distractor condition separately and for the same nine subjects that were included in the analyses described above (on latency error saccades to distractor). Both $t$ tests showed that the mean latency of error saccades directed to the distractor was shorter than of error saccades directed elsewhere. When the CS- distractor was presented, mean latency of error saccades to the CS- distractor (195 ms, $S E=5 \mathrm{~ms}$ ) was shorter than error saccades directed elsewhere $(237 \mathrm{~ms}, S E=7 \mathrm{~ms}), t(8)=7.54, p$ $<.01, d=2.7$, and when the CS+ distractor was presented mean latency of error saccades directed to the CS+ distractor $(193 \mathrm{~ms}, S E=5 \mathrm{~ms})$ was shorter than error saccades directed elsewhere (239 ms, $S E=5 \mathrm{~ms}), t(8)=$ $6.2, p<.01, d=2.5$. The shorter latency of error saccades directed to the distractors relative to error saccades directed elsewhere indicates that the oculomotor capture saccades were indeed driven by the information at that location and were not driven by a nonspecific enhanced state anxiety induced by the distractors. 


\section{Discussion}

Results from the current study show that a threatening stimulus modulates attentional and visual selection in a bottom-up driven manner, indicating automatic prioritization of threatening stimuli in the visual system. Mean latency of saccades directed to a neutral target in the presence of a threatening distractor was increased relative to the nonthreatening distractor and to no distractor conditions, indicating exogenous attentional capture and delayed disengagement from the threatening distractor. Moreover, the threatening distractor captured the eyes more often than the nonthreatening distractor, indicating automatic modulation of oculomotor capture.

\section{Attentional capture and disengagement}

An increase in saccade latency to a target in the presence of a distractor is previously explained in the context of the premotor theory of attention (Rizzolatti et al., 1987). The premotor theory of attention argues that spatial attention and saccade programming are highly intertwined and suggests that a shift of spatial attention to a location automatically activates the oculomotor system to program a saccade to that location (but see Belopolsky \& Theeuwes, 2012). Therefore, in the current study, the increase in saccade latency in the presence of a threatening distractor could have resulted from the extra time it took to reprogram a saccade from the distractor to the target (Godijn \& Theeuwes, 2002). In this scenario, covert attention is first captured by the salient threatening distractor before attention is shifted to the target (as in Schmidt et al., 2014). This account is consistent with results from previous cueing studies. In these studies, an eye movement had to be made to one of two target locations that were previously cued with threatening or nonthreatening stimuli (Bannerman et al., 2010a, 2010b; Schmidt, Belopolsky, \& Theeuwes, 2015). Both studies showed the tight coupling between a shift of spatial attention to a threatening stimulus and the direct influence on the oculomotor system. For example, Bannerman et al. (2010a, 2010b) showed that a briefly presented threatening peripheral cue decreased saccade latency relative to a nonthreatening cue when an eye movement had to be executed to a target presented at the same location (valid cue) and increased saccade latency when the target was presented at the opposite location (invalid cue). Likewise, Schmidt et al. (2015) showed that a peripherally presented threatening stimulus interfered with subsequent endogenously driven saccades; a decrease in saccade latency when a saccade had to be executed to the location previously cued with a threatening stimulus relative to a nonthreatening stimulus, and an increase in saccade latency when a saccade had to be executed away from that location.
Furthermore, simple prosaccade tasks demonstrated the influence of covert emotional attention on the oculomotor system by measuring the trajectory of a saccade. In a prosaccade task, participants are asked to make a saccade to a neutral target stimulus while simultaneously a sudden onset distractor is presented next to the saccadic path. Typically, fast saccades curve toward a distractor and slower saccades curve away from a distractor (Van der Stigchel, Meeter, \& Theeuwes, 2006). Previous studies found that the deviations of the saccade trajectory-towards as well as away from the distractor - are increased in the presence of a threatening distractor relative to a nonthreatening distractor (e.g., McSorley \& Van Reekum, 2013; Mulckhuyse et al., 2013; Nummenmaa, Hyönä, \& Calvo, 2009; Schmidt et al., 2012; but see West, Al-Aidroos, Susskind, \& Pratt, 2011). These modulations of saccade deviation indicate that the threatening distractor captured and held attention and thereby affected the trajectory of a saccade (Van der Stigchel et al., 2006).

The eye movement studies described above do not only evidence the strong link between spatial attention and saccade programming, they also demonstrate that threatening stimuli capture and hold attention, which is consistent with our findings. However, the cumulative distributions also showed that the fastest goal-driven saccades were not modulated by threat. In other words, the threatening distractor presumably did not capture attention in these trials. This implies that a threatening distractor may not always be automatically prioritized by the visual system and can be ignored, possibly by exerting goaldriven control (Leber, 2010).

\section{Oculomotor capture}

The oculomotor capture trials indicate that in some trials the onset distractor captured not only covert attention but also the eyes. These error saccades are typically very fast eye movements and explained by faster bottom-up activation of the distractor location relative to slower top-down activation of the target location in a common saccade map (Godijn \& Theeuwes, 2002). The common saccade map is sometimes referred to as a priority map (Fecteau \& Munoz, 2006) that integrates stimulus-related with task-related or behavioralrelevant information. Our results show that more error saccades were directed toward the threatening distractor than to the nonthreatening distractor, indicating that threat modulates this automatically driven process. However, there was no latency difference between error saccades directed to the threatening and the nonthreatening distractors. This may suggest that threatening information did not reach the saccade map earlier than the nonthreatening information. In other words, this may suggest that threat is not processed faster. Instead, it may indicate that the distractor-related activity in the saccade map was strengthened by the threatening information, and therefore the threshold to make a saccade to that location 
was more often reached, resulting in more but not per se faster oculomotor capture.

Oculomotor capture has not been investigated before with task-irrelevant emotional distractor stimuli, although Mulckhuyse et al. (2013) found some evidence of oculomotor capture by task-irrelevant emotional distractors in a prosaccade task. However, that study did not measure latency of the error saccades. Other studies, using free viewing paradigms or forced choice tasks, measured proportion of first fixation as an index of oculomotor capture (e.g., Calvo \& Lang, 2004; Calvo, Avero, \& Lundqvist, 2006; Humphrey et al., 2012; Nummenmaa et al., 2006). Only a few studies reported faster saccades to threatening than to nonthreatening stimuli (Bannerman, Milders, de Gelder, \& Sahraie, 2009; Bannerman, Milders, \& Sahraie, 2009; LoBue et al., 2014; Reynolds, Eastwood, Partanen, Frischen, \& Smilek, 2009; but see Calvo et al., 2006; Derakshan \& Koster, 2010; Mogg, Millar, \& Bradley, 2000), suggesting faster processing of threatening stimuli. However, in these studies the threatening stimulus was always task relevant. For example, in LoBue et al. (2014), participants had to judge whether a matrix of pictures were of the same category or if one of the pictures was of another category (as in Öhman, Flykt, \& Esteves, 2001). Therefore, participants had to attend to the pictures in order to perform the task. Possibly, threat affects saccade latency only when the threatening stimulus is to some extent task relevant and thus part of the top-down goal or task set (as in Bannerman, Milders, de Gelder, \& Sahraie, 2009; Bannerman, Milders, \& Sahraie, 2009; Kissler \& Keil, 2008; LoBue et al., 2014; Nummenmaa et al., 2006). Our results show that when a threatening stimulus captures the eyes in a bottom-up driven manner, saccade latency is unaffected. Further research is needed to disentangle the influence of threat on these topdown and bottom-up driven processes in visual selection.

\section{Underlying neural mechanisms}

What may be the underlying neural mechanisms of the influence of threat on the oculomotor system? The most important areas and structures involved in oculomotor behavior are the brainstem, the superior colliculus (SC), the pulvinar, and the parietal and frontal eye fields (PEF and FEF; e.g., Munoz, 2002; Schall, 1995; Shipp, 2004).The SC, a midbrain structure, is viewed as the neural locus of the common saccade map where bottom-up and top-down information converge (Godijn \& Theeuwes, 2002; Trappenberg, Dorris, Munoz, \& Klein, 2001). The SC receives visual information directly from the retina and indirectly from visual cortex and integrates incoming visual information with motor output (Robinson \& McClurkin, 1989). In addition, the SC is also part of the socalled specialized subcortical pathway, as suggested by the direct route theory (e.g., De Gelder et al., 2011; LeDoux, 2000, 2003; Öhman, Flykt, \& Esteves, 2001). It is supposed to transfer threatening visual information via the pulvinar to the amygdala, a structure involved in processing of emotional stimuli and in the expression of conditioned fear (Davidson \& Irwin, 1999; Davis, 1992a, 1992b; Davis \& Whalen, 2001; Vuilleumier, 2005). Moreover, the subcortical pathway is also believed to be involved in fear conditioning (Hamm et al., 2003; LeDoux, 2000). If prioritization of threatening visual information is accomplished via a direct route to the amygdala (LeDoux, 2000, 2003; Vuilleumier, 2005), reciprocal connections between the SC, pulvinar, and amygdala (Linke, De Lima, Schwegler, \& Pape, 1999; Morris, Ohman, \& Dolan, 1999; Tamietto \& De Gelder, 2010) may have enhanced the representation of the threatening distractor location in $\mathrm{SC}$, resulting in more attentional and oculomotor capture (Godijn $\&$ Theeuwes, 2002). However, the direct route theory also suggests that threatening stimuli are processed faster than nonthreatening stimuli. Consequently, this would result in faster eye movements to threatening than nonthreatening stimuli (but see West et al., 2011). Nonetheless, in our study, latencies of error saccades to the distractors were not affected by threat, indicating that the threatening distractor was not processed faster than the nonthreatening distractor.

The multiple-waves model suggests that prioritization of emotional visual information is accomplished not via one direct route but via modulating interactions between subcortical and cortical connections (Pessoa \& Adolphs, 2010). In this model, the pulvinar plays a more substantial role in signifying emotional saliency than the amygdala. The pulvinar is associated with saccadic behavior (Robinson \& McClurkin, 1989) and with processing of behavioral-salient relevant visual stimuli (Pessoa \& Adolphs, 2010; Robinson \& Petersen, 1992; Shipp, 2004). It has connections with parietal and frontal areas, which are involved in salience detection and activation of possible target locations (McPeek, 2006; Walker, Techawachirakul, \& Haggard, 2009), in spatial attention (Shipp, 2004), and oculomotor behavior (Munoz, 2002). Together with the SC and the pulvinar, the FEF and PEF (the latter is also referred to as lateral intraparietal area; LIP) are thought to be involved in the construction of a so-called saliency map (Munoz, 2002; Thompson \& Bichot, 2005) or priority map (Fecteau \& Munoz, 2006). Modulating interactions from the pulvinar might have enhanced or strengthened the representation of the threatening distractor location in this priority map. As a result of the enhanced representation in the oculomotor system, the threatening distractor was selected more often than the nonthreatening distractor. In sum, our findings suggest that threatening stimuli are automatically prioritized in visual selection, albeit not, per se, faster processed.

Acknowledgments This research was funded by a VENI grant from NWO (Netherlands Organization for Scientific Research) to M. M. E. S. D. was supported through a European Commission Marie Curie ITN grant (606901). 


\section{References}

Bannerman, R. L., Milders, M., de Gelder, B., \& Sahraie, A. (2009). Orienting to threat: Faster localization of fearful facial expressions and body postures revealed by saccadic eye movements. Proceedings of the Royal Society B: Biological Sciences, 276, 1635-1641.

Bannerman, R. L., Milders, M., \& Sahraie, A. (2009). Processing emotional stimuli: Comparison of saccadic and manual choice-reaction times. Cognition and Emotion, 23, 930-954.

Bannerman, R. L., Milders, M., \& Sahraie, A. (2010a). Attentional bias to brief threat-related faces revealed by saccadic eye movements. Emotion, 10, 733-738.

Bannerman, R. L., Milders, M., \& Sahraie. (2010b). Attentional cueing: Fearful body postures capture attention with saccades. Journal of Vision, 10, 23.

Belopolsky, A. V., \& Theeuwes, J. (2012). Updating the premotor theory: The allocation of attention is not always accompanied by saccade preparation. Journal of Experimental Psychology. Human Perception and Performance, 38(4), 902-914.

Boddez, Y., Baeyens, F., Luyten, L., Vansteenwegen, D., Hermans, D., \& Beckers, T. (2013). Rating data are underrated: Validity of US expectancy in human fear conditioning. Journal of Behavior Therapy and Experimental Psychiatry, 44, 201-206.

Brown, J. S., Kalish, H., \& Farber, I. E. (1951). Conditioned fear as revealed by magnitude of startle response to an auditory stimulus. Journal of Experimental Psychology, 41, 317-328.

Calvo, M. G., Avero, P., \& Lundqvist, D. (2006). Facilitated detection of angry faces: Initial orienting and processing efficiency. Cognition and Emotion, 20(6), 785-811.

Calvo, M. G., \& Lang, P. J. (2004). Gaze patterns when looking at emotional pictures: Motivationally biased attention. Motivation and Emotion, 28, 221-243.

Calvo, M. G., \& Nummenmaa, L. (2008). Detection of emotional faces: Salient physical features guide effective visual search. Journal of Experimental Psychology: General, 137(3), 471-494.

Carretié, L. (2014). Exogenous (automatic) attention to emotional stimuli: A review. Cognitive, Affective, \& Behavioral Neuroscience. doi:10. 3758/s13415-014-0270-2

Dalmaijer, E. S., Mathôt, S., \& Van der Stigchel, S. (2014). PyGaze: An open-source, cross-platform toolbox for minimal-effort programming of eye-tracking experiments. Behavior Research Methods, 46, 913-921.

Davidson, R. J., \& Irwin, W. (1999). The functional neuroanatomy of emotion and affective style. Trends in Cognitive Sciences, 3(1), 1121.

Davis, M. (1992a). The role of the amygdala in conditioned fear. In J. P. Aggleton (Ed.), The amygdala (pp. 255-306). Wilmington, DE: Wiley-Liss.

Davis, M. (1992b). The role of the amygdala in fear and anxiety. Annual Review of Neuroscience, 15, 353-375.

Davis, M., \& Whalen, P. J. (2001). The amygdala: Vigilance and emotion. Molecular Psychiatry, 6(1), 13-34.

De Gelder, B., Van Honk, J., \& Tamietto, M. (2011). Emotion in the brain: Of low roads, high roads and roads less travelled. Nature Reviews. Neuroscience, 11, 773-783.

Delgado, M. R., Olsson, A., \& Phelps, E. A. (2006). Extending animal models of fear conditioning to humans. Biological Psychology, 73, 39-48.

Derakshan, N., \& Koster, E. H. W. (2010). Processing efficiency in anxiety: Evidence from eye-movements during visual search. Behaviour Research and Therapy, 48, 1180-1185.

Fecteau, J. H., \& Munoz, D. P. (2006). Salience, relevance, and firing: A priority map for target selection. Trends in Cognitive Sciences, 10, 382-390.
Fox, E., Russo, R., Bowles, R., \& Dutton, K. (2001). Do threatening stimuli draw or hold visual attention in subclinical anxiety? Journal of Experimental Psychology. General, 130(4), 681-700.

Fox, E., Russo, R., \& Dutton, K. (2002). Attentional bias for threat: Evidence for delayed disengagement from emotional faces. Cognition and Emotion, 16, 355-379.

Godijn, R., \& Theeuwes, J. (2002). Programming of endogenous and exogenous saccades: Evidence for a competitive integration model. Journal of Experimental Psychology. Human Perception and Performance, 28(5), 1039-1054.

Hamm, A. O., Weike, A. I., Schupp, H. T., Treig, T., Dressel, A., \& Kessler, C. (2003). Affective blindsight: Intact fear conditioning to a visual cue in a cortically blind patient. Brain, 126, 267-275.

Hansen, C., \& Hansen, R. (1988). Finding the face in the crowd: An anger superiority effect. Journal of Personality and Social Psychology, 54, 917-924.

Hofmann, S. G. (2008). Cognitive processes during fear acquisition and extinction in animals and humans: Implications for exposure therapy of anxiety disorders. Clinical Psychology Review, 28, 199-210.

Humphrey, K., Underwood, G., \& Lambert, T. (2012). Salience of the lambs: A test of the saliency map hypothesis with pictures of emotive objects. Journal of Vision, 12(1). doi:10.1167/12.1.22

Hunt, A. R., Cooper, R. M., Hungr, C., \& Kingstone, A. (2007). The effect of emotional faces on eye movements and attention. Visual Cognition, 15(5), 513-531.

Kissler, J., \& Keil, A. (2008). Look-don't look! How emotional pictures affect pro- and anti saccades. Experimental Brain Research, 188, $215-222$.

Lang, P. J., Greenwald, M. K., Bradley, M. M., \& Hamm, A. O. (1993). Looking at pictures: Affective, visceral, and behavioral reactions. Psychophysiology, 30, 261-273.

Leber, A. B. (2010). Neural predictors of within-subject fluctuations in attentional control. The Journal of Neuroscience, 30, 11458-11465.

LeDoux, J. E. (2000). Emotion circuits in the brain. Annual Review of Neuroscience, 23, 155-184.

LeDoux, J. (2003). The emotional brain, fear, and the amygdala. Cellular and Molecular Neurobiology, 23(4/5), 727-738.

LeDoux, J. E. (2014). Coming to terms with fear. PNAS, 11, 2871-2878.

Liddell, B. J, Brown, K. J., Kemp, A. H, Barton, M. J., Das, P., Peduto, A., . . Williams, L. M. (2005). A direct brainstem-amygdala-cortical "alarm" system for subliminal signals of fear. NeuroImage, 24, 235-243.

Linke, R., De Lima, A. D., Schwegler, H., \& Pape, H. C. (1999). Direct synaptic connections of axons from the superior colliculus with identified thalamo-amygdaloid projection neurons in the rat: Possible substrates of a subcortical visual pathway to the amygdala. Journal of Comparative Neurology, 403, 158-170.

Lissek, S., Biggs, A. L., Rabin, S. J., Cornwell, B. R., Alvarez, R. P., Pine, D. S., \& Grillon, C. (2008). Generalization of conditioned fearpotentiated startle in humans: Experimental validation and clinical relevance. Behaviour Research and Therapy, 46(5), 678-687.

LoBue, V., Matthews, K., Harvey, T., \& Stark, S. L. (2014). What accounts for the rapid detection of threat? Evidence for an advantage in perceptual and behavioral responding from eye movements. Emotion, 14, 816-823.

Mackintosh, N. J. (1983). Conditioning and associative learning. Oxford, England: Oxford University Press.

Maren, S. (2001). Neurobiology of Pavlovian fear conditioning. Annual Review of Neuroscience, 24, 897-931.

McPeek, R. M. (2006). Incomplete suppression of distractor-related activity in the frontal eye field results in curved saccades. Journal of Neurophysiology, 96, 2699-2711.

McSorley, E., Haggard, P., \& Walker, R. (2006). Time course of oculomotor inhibition revealed by saccade trajectory modulation. Journal of Neurophysiology, 96, 1420-1424. 
McSorley, E., \& Van Reekum, C. M. (2013). The time course of implicit affective picture processing: An eye movement study. Emotion, 13, 769-773.

Mogg, K., Millar, N., \& Bradley, B. P. (2000). Biases in eye movements to threatening facial expressions in generalized anxiety disorder and depressive disorder. Journal of Abnormal Psychology, 109, 695704.

Morris, J. S., Ohman, A., \& Dolan, R. J. (1999). A subcortical pathway to the right amygdala mediating "unseen" fear. Proceedings of the National Academy of Sciences of the United States of America, 96, 1680-1685.

Mulckhuyse, M., Crombez, G., \& Van der Stigchel, S. (2013). Conditioned fear modulates visual selection. Emotion, 13(3), 529536.

Mulckhuyse, M., Van Zoest, W., \& Theeuwes, J. (2008). Capture of the eyes by relevant and irrelevant onsets. Experimental Brain Research, 186, 225-235.

Munoz, D. P. (2002). Saccadic eye movements: Overview of neural circuitry. Progress in Brain Research, 140, 89-96.

Notebaert, L., Crombez, G., Van Damme, S., De Houwer, J., \& Theeuwes, J. (2011). Signals of threat do not capture, but prioritize, attention: A conditioning approach. Emotion, 11(1), 81.

Nummenmaa, L., Hyönä, J., \& Calvo, M. G. (2006). Eye movement assessment of selective attentional capture by emotional pictures. Emotion, 6, 257-268.

Nummenmaa, L., Hyönä, J., \& Calvo, M. G. (2009). Emotional scene content drives the saccade generation system reflexively. Journal of Experimental Psychology. Human Perception and Performance, 35(2), 305-323.

Öhman, A., Flykt, A., \& Esteves, F. (2001). Emotion drives attention: Detecting the snake in the grass. Journal of Experimental Psychology. General, 130(3), 466-478.

Öhman, A., Lundqvist, D., \& Esteves, F. (2001). The face in the crowd revisited: A threat advantage with schematic stimuli. Journal of Personality and Social Psychology, 80, 381-396.

Pessoa, L., \& Adolphs, R. (2010). Emotion processing and the amygdala: From a 'low road' to 'many roads' of evaluating biological significance. Nature Review Neuroscience, 11, 773-783.

Pineles, S. L., Orr, M. R., \& Orr, S. P. (2009). An alternative scoring method for skin conductance responding in a differential fear conditioning paradigm with a long-duration conditioned stimulus. Psychophysiology, 46, 1-12.

Ratcliff, R. (1979). Group reaction-time distributions and an analysis of distribution statistics. Psychological Bulletin, 86(3), 446-461.

Reynolds, M. G., Eastwood, J. D., Partanen, M., Frischen, A., \& Smilek, D. (2009). Monitoring eve movements while searching for affective faces. Visual Cognition, 17, 318-333.

Rizzolatti, G., Riggio, L., Dascola, I., \& Umilta, C. (1987). Reorienting attention across the horizontal and vertical meridians: Evidence in favor of a premotor theory of attention. Neuropsychologia, 25(1A), 31-40.

Robinson, D. L., \& McClurkin, J. W. (1989). The visual superior colliculus and pulvinar. In R. H. Wurtz \& M. E. Goldberg (Eds.),
The neurobiology of saccadic eye movements (pp. 337-360). Amsterdam, The Netherlands: Elsevier.

Robinson, D. L., \& Petersen, S. E. (1992). The pulvinar and visual salience. Trends in Neurosciences, 15, 127-132.

Schall, J. D. (1995). Neural basis of saccade target selection. Reviews in the Neurosciences, 6(1), 63-85.

Schmidt, L. J., Belopolsky, A. V., \& Theeuwes, J. (2012). The presence of threat affects saccade trajectories. Visual Cognition, 20, 284-299.

Schmidt, L. J., Belopolsky, A. V., \& Theeuwes, J. (2014). Attentional capture by signals of threat. Cognition and Emotion. doi:10.1080/ 02699931.2014 .924484

Schmidt, L. J., Belopolsky, A. V., \& Theeuwes, J. (2015). Potential threat attracts attention and interferes with voluntary saccades. Emotion, 15(3), 329-338.

Sehlmeyer, C., Schoning, S., Zwitserlood, P., Pfleiderer, B., Kircher, T., Arolt, V., \& Konrad, C. (2009). Human fear conditioning and extinction in neuroimaging: A systematic review. PloS One, 4, e5865.

Shipp, S. (2004). The brain circuitry of attention. Trends in Cognitive Science, 8, 223-230.

Tamietto, M., \& De Gelder, B. (2010). Neural basis of non-conscious perception of emotional signals. Nature Reviews Neuroscience, 11 687-709.

Theeuwes, J. (2010). Top-down and bottom-up control of visual selection. Acta Psychologica, 135, 77-99.

Theeuwes, J., Kramer, A. E., Hahn, S., \& Irwin, D. E. (1998). Our eyes do not always go where we want them to go: Capture of the eyes by new objects. Psychological Science, 9, 379-385.

Thompson, K. G., \& Bichot, N. P. (2005). A visual salience map in the primate frontal eye field. Progress in Brain Research, 147, 249-262.

Tipples, J., Young, A. W., Quinlan, P., Broks, P., \& Ellis, A. W. (2002). Searching for threat. The Quarterly Journal of Experimental Psychology, 55A, 1007-1026.

Trappenberg, T. P., Dorris, M. D., Munoz, D. P., \& Klein, R. M. (2001). A model of saccade initiation based on the competitive integration of exogenous and endogenous signals in the superior colliculus. Journal of Cognitive Neuroscience, 13, 256-271.

Van der Stigchel, S., Meeter, M., \& Theeuwes, J. (2006). Eye movement trajectories and what they tell us. Neuroscience \& Biobehavioral Reviews, 30, 666-679.

Vuilleumier, P. (2005). How brains beware: Neural systems for emotional attention. Trends in Cognitive Sciences, 9(12), 585-594.

Walker, R., Techawachirakul, P., \& Haggard, P. (2009). Frontal eye field stimulation modulates the balance of salience between target and distractors. Brain Research, 270, 54-63.

West, G. L., Al-Aidroos, N., Susskind, J., \& Pratt, J. (2011). Emotion and action: The effect of fear on saccadic performance. Experimental Brain Research, 209, 153-158.

Yantis, S. (1993). Stimulus-driven attentional capture and attentional control settings. Journal of Experimental Psychology: Human Perception and Performance, 19, 676-681. 